\title{
Gillespie.jl: Stochastic Simulation Algorithm in Julia
}

\section{Simon DW Frost ${ }^{1}$}

DOI: $10.21105 /$ joss. 00042

\section{Software}

- Review ¿

- Repository ca

- Archive ${ }^{\top}$

\section{Licence}

Authors of JOSS papers retain copyright and release the work under a Creative Commons Attribution 4.0 International License (CC-BY).
1 University of Cambridge

\section{Summary}

Gillespie.jl (Frost 2016) is a Julia package for stochastic simulation using Gillespie's direct method (sometimes called the Doob-Gillespie algorithm) (Doob 1945; Gillespie 1977), an approach widely used in many fields, including systems biology and epidemiology. It borrows the basic interface (although none of the code) from the R library GillespiesSA by Mario Pineda-Krch (Pineda-Krch 2008), although Gillespie.jl only implements the standard exact method at present, whereas GillespieSSA also includes other methods, such as tau-leaping, etc.. Gillespie.jl is intended to offer performance on par with hand-coded $\mathrm{C}$ code, while maintaining a simple but flexible interface.

\section{References}

Doob, Joseph L. 1945. "Markoff Chains-Denumerable Case." Transactions of the American Mathematical Society 58 (3). JSTOR: 455-73. doi:10.2307/1990339.

Frost, Simon D.W. 2016. "Gillespie.jl: Stochastic Simulation in Julia." https://github. com/sdwfrost/Gillespie.jl.

Gillespie, Daniel T. 1977. "Exact Stochastic Simulation of Coupled Chemical Reactions." The Journal of Physical Chemistry 81 (25). ACS Publications: 2340-61. doi:10.1021/j100540a008.

Pineda-Krch, Mario. 2008. "GillespieSSA: Stochastic Simulation Algorithm in R." Journal of Statistical Software 25: 12. doi:10.18637/jss.v025.i12. 